\title{
Treatment length as a determinant of immunization against learned helplessness in humans
}

\author{
JACK R. NATION and LEE G. BOYAJIAN \\ Texas A\&M University, College Station, Texas 77843
}

\begin{abstract}
Following the induction of learned helplessness via forced exposure to recurring failure, college students experienced either abbreviated ( 35 trials) or extended (70 trials) treatment with either partial reinforcement or continuous reinforcement during a reversal phase. Subsequently, all subjects encountered a second noncontingency involving a protracted period of nonreinforcement (extinction). The results of the experiment indicated that partial as contrasted with continuous reinforcement occasioned greater immunization against recurrent helplessness; that is, subjects treated with intermittent success and failure were more persistent in the face of repeated exposures to negative outcomes than were subjects treated with success only. Moreover, extended treatment resulted in greater immunization against helplessness than did abbreviated treatment, regardless of schedule type. These findings are discussed within the conceptual frameworks of frustration theory and the reformulated learned helplessness hypothesis.
\end{abstract}

One concern in the recent learned helplessness literature (see Abramson, Seligman, \& Teasdale, 1978) has been the isolation of a treatment routine that effectively reverses helplessness and simultaneously immunizes the person against recurring uncontrollability. Along these lines, Nation and Massad (1978) have suggested the use of intermittent reinforcement schedules for the alleviation of helplessness effects. The basic idea here is that partial, as contrasted with continuous, schedules of reinforcement operate to foster greater tolerance for such negative outcomes as failure or nonreinforcement and thereby promote persistence in a helplessness-inducing milieu. Indeed, previous studies provide empirical confirmation of the superior potential associated with the use of intermittent reinforcement as a reversal technique (e.g., Jones, Nation, \& Massad, 1977; Nation, Cooney, \& Gartrell, 1979).

Two opponent theoretical rationales offer explanations of the partial reinforcement effects mentioned above. On one hand, the reformulated learned helplessness theory (Abramson et al., 1978) views the increased tolerance for uncontrollability common to the use of partial reinforcement in a reversal phase as consequential to the formation of an attribution for failure outcomes to unstable factors (Seligman, Note 1). That is, intermittent success and failure experiences may foster an expectancy that reinforcement does occur eventually with the continued production of relevant behaviors.

We would like to thank John Cooney for his unselfish assistance in analyzing the data. Requests for reprints should be sent to Jack R. Nation, Texas A\&M University, College Station, Texas 77843 .
Alternatively, the enhanced persistence effects resulting from partial reinforcement experiences may be due to an Amsel-type frustration effect; that is, through counterconditioning, the frustrative cues arising out of the emotions produced by failure come to mediate an attitude of approach and ultimately persistence in the face of recurring setbacks (see Nation \& Massad, 1978, for a detailed account of this frustration model).

The aim of the present helplessness study is to sharpen this theoretical picture concerned with the increased immunization benefits associated with partial reinforcement schedules. Specifically, treatment lengths and schedules were manipulated in a parametric analysis, yielding contrasting theoretical predictions. The reformulated learned helplessness model forecasts greater immunization against the effects of a situational noncontingency (recurring nonrewards) with increasing lengths of treatment, regardless of the precise nature of the reinforcement schedules (partial or continuous). Ostensibly, the greater the treatment length, the greater is the solidity of the attributions promoting persistence (viz., an internal, stable attribution for success following continuous reinforcement and an unstable attribution for failure following partial reinforcement). In contrast, extended treatment should lessen the immunization against a recurring period of failure (helplessness) from the vantage of frustration theory, particularly with continuous reinforcement. This prediction derives from the "overtraining extinction effect," which with animals occurs when abbreviated training produces greater resistance to extinction than does extended acquisition training. This phenomenon has been observed in the infrahuman laboratory on numerous occasions (e.g., 
Traupmann, 1972) and is viewed as consistent with frustration theory (see Nation \& Woods, 1980). So, as a formal test of these quite different theoretical perspectives, college students experienced the inducement of helplessness, reversal training with either an abbreviated or extended session of continuous or partial reinforcement, and, finally, a second period of uncontrollability (extinction).

\section{METHOD}

\section{Subjects}

Subjects were 40 female and 40 male introductory psychology students at Texas A\&M University who participated in partial fulfillment of course requirements.

\section{Apparatus and Material}

Helplessness induction. A series of six five-dimensional concept identification problems served as stimulus materials. The problems involved stimuli identical to those detailed in the pretreatment phase of an earlier study (Nation et al., 1979).

Treatment. The apparatus for the treatment phase was the human shuttlebox described for the therapy phase (Phase 2: task-dissimilar condition) of the Nation et al. (1979) study. Reinforcement (tone termination), schedule conditions (100\% or $50 \%$ ), and other programming considerations were as detailed in that earlier experiment.

Persistence test. The apparatus used during the persistence test phase was the same shuttle used earlier for treatment. During this phase, a selector switch was set so that tone termination was not possible.

Mood scales. Three 10-point scales were used to provide an index of sadness, frustration, and personal competency.

\section{Procedure}

All subjects completed the mood scales when they first arrived at the laboratory and again following each of three phases.

Helplessness induction. The procedure during the helplessness induction phase was the same for the no-treatment abbreviated group (NoT-AB) and the no-treatment extended group (NoT-EX). Subjects in both groups were not exposed to success or failure during this initial phase but simply looked passively at the cards for $5 \mathrm{sec} / \mathrm{card}$, a time approximating the normal time spent per card by the other groups. Both the soluble abbreviated group (SOL-AB) and the soluble extended group (SOL-EX) were required to learn six soluble problems during this phase. The six different problems were presented in blocks of 10 trials each.

Additionally, two abbreviated groups received insoluble problems during the helplessness-induction phase. Both insoluble continuous reinforcement subjects (IN-CRF-AB) and insoluble partial reinforcement subjects (IN-PRF-AB) received six completely insoluble problems in this initial phase. Procedural events (noncontingent feedback, instructions, number of trials on each problem, etc.) were identical to those procedures described for insoluble subjects in the Nation et al. (1979) study. Two extended groups (IN-CRF-EX, IN-PRF-EX) experienced the identical insoluble helplessness-induction contingencies used for Groups IN-CRF-AB and IN-PRF-AB.

Treatment. All subjects performed on the human shuttle task. Subjects in the abbreviated conditions (NoT-AB, SOL-AB, IN-CRF-AB, IN-PRF-AB) were administered only 35 trials in the treatment phase. Conversely, subjects in the extended conditions (NoT-EX, SOL-EX, IN-CRF-EX, IN-PRF-EX) were administered a total of 70 trials in the treatment phase. In every case, each trial was signaled by the onset of a 5 -sec red warning light (CS). At the end of the 5-sec avoidance interval, a tone (UCS) came on for a maximum duration of $6.5 \mathrm{sec}$, after which the stimuli offset together. The intertrial interval for the tones ranged from 6.5 to $20 \mathrm{sec}$, with a mean of $16.9 \mathrm{sec}$. The first five trials of treatments were continuously reinforced trials for all subjects and involved movement of the shuttle peg to the far left position. Thus, subjects in all eight groups could terminate the tone on the initial five trials. The remaining trials differed according to selected treatment schedules given to each group. Groups NoT-AB, SOL-AB, and IN-CRF-AB continued to operate under continuous reinforcement contingencies, as did Groups NoT-EX, SOL-EX, and IN-CRF-EX; that is, criterion responding resulted in termination of the tone. In contrast, Group IN-PRF-AB and Group IN-PRF-EX experienced a partial reinforcement schedule during treatment and could terminate the tone on only $50 \%$ of the trials. On nonreinforced trials, the tone would not terminate, regardless of the number of shuttle responses performed. On reinforced trials, moving the peg to the far left terminated the tone in a manner similar to the continuously reinforced groups.

The dependent measures obtained on the shuttle task were latency (defined as the latency from trial onset to completion of the criterion behavior), number of failures (number of trials on which subjects failed to make the criterion response), and number of responses (the number of times the subject made the criterion response during a given trial).

Persistence test. Upon completion of the treatment training, subjects in all eight groups were given 90 nonreinforced (extinction) trials. The same dependent measures used for treatment were used again for the persistence test.

\section{RESULTS}

\section{Mood Data}

The analyses performed on the mood data showed a high degree of consistency across dependent measures. On all measures, insolubility during pretreatment had the effect of inducing negative affect; subsequent treatment, regardless of type, successfully reversed these negative feelings, but negative affect was again occasioned by insolubility during the persistence test.

Mood was analyzed by separate repeated-measures analysis of variance (ANOVA) tests performed on levels of reported sadness, frustration, and personal competency. The most important results came from the reports of significant Conditions by Phases interaction tests for sadness $[F(9,192)=3.20, p<.01]$, frustration $[F(9,192)=4.96, p<.01]$, and personal competency $[F(9,192)=6.28, p<.01]$. Newman-Keuls post hoc comparisons revealed the following significant $(p<.05)$ trends: Although not originally different, the insoluble conditions showed greater negative affect than did the no-treatment or soluble conditions following pretreatment; these differences were removed by the treatment manipulations, and all conditions showed increases in negative affect following the persistence test. None of the relevant interactions with length of treatment was found to reach acceptable levels for statistical significance.

\section{Treatment}

The analyses of the treatment data revealed that the insoluble pretreatment manipulation was effective with respect to inducing helplessness (behavioral deficits). By the end of treatment, exposure to repeated success experiences had completely reversed these helplessness effects. Neither schedule type nor length of treatment 
produced differential performances at the completion of treatment.

Because all three dependent measures showed similar results, and to save space, statistical confirmation of the above is limited to the use of the failure measure. Of particular interest was the finding of a significant Condition by Block interaction effect $[F(3,64)=30.01$, $\mathrm{p}<.01]$. Post hoc comparisons indicated that on the first block of five trials, the two insoluble conditions that did not differ were each inferior (made more failures) relative to the no-treatment and soluble conditions that were not different. By the last five-trial block of treatment, the insoluble conditions had improved to the point that there were no differences across conditions.

\section{Persistence Test}

The analyses of the persistence data showed rather dramatically that partial reinforcement during treatment produced greater immunization against a recurring noncontingency than did continuous reinforcement. Moreover, it was revealed that treatment length was positively related to immunization; that is, for both continuous and partial schedules, extended treatment occasioned greater persistence in the face of repeated nonreinforcement experiences than did abbreviated treatment.

Statistical confirmation of the above was made available via separate repeated-measures ANOVAs performed on the latency, failure, and number of responses measures. Once again, the observed effects were quite uniform across measures, with the strongest effects occurring on the failure variable. Accordingly, reporting of the persistence test data is restricted here to the failure measure.
The most important result was the report of a significant Condition by Length of Treatment by Five-Trial Block interaction test $[\mathrm{F}(51,1087)=2.37, \mathrm{p}<.01]$. The means of this interaction are graphically depicted in Figure 1. Post hoc analyses of these cell means showed that the two groups that had experienced partial reinforcement treatment (IN-PRF-AB, IN-PRFEX) were superior to all other groups, with significant separation beginning on Block 3. Furthermore, it was revealed that extended treatment produced greater persistence than did abbreviated treatment, regardless of schedule. The separation between IN-PRF-EX subjects and IN-PRF-AB subjects was first evident on Block 10 and continued for the duration of the persistence test. The superiority of Group IN-CRF-EX relative to Group IN-CRF-AB began as early as the second block, as did the superiority of Group SOL-EX relative to Group SOL-AB. Differential performances in favor of Group NoT-EX when compared with Group NoT-AB began to emerge by Block 3 .

\section{DISCUSSION}

The findings from this study show that length of helplessness reversal training, regardless of schedule characteristics, is positively related to immunization against future insoluble situations. While there was no evidence that any treatment manipulation had a prophylactic effect regarding the induction of negative mood states, substantially greater behavioral persistence in the face of aversive recurring outcomes was evidenced for subjects given extended reversal training compared with subjects given abbreviated reversal training. The failure to observe immunization effects with mood is perhaps relatively less important than the behavioral results, for it should be understood that an attitude of persistence that ultimately renders a person more facile when environmental conditions finally do improve will alter negative affect in any case.

The data described herein are compatible with the predic-

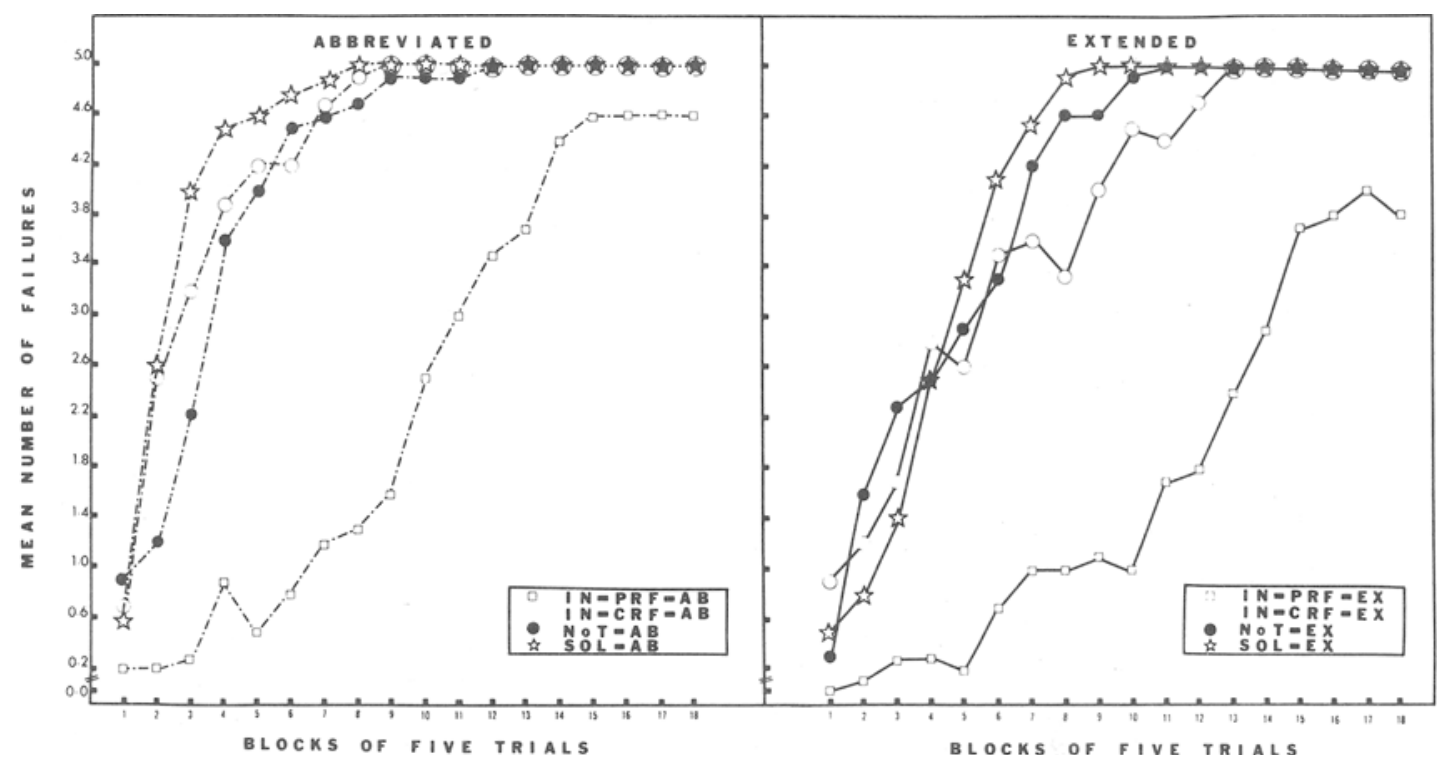

Figure 1. Mean number of failures for the four abbreviated treatment groups (IN-PRF-AB, IN-CRF-AB, NoT-AB, SOL-AB) and the four extended treatment groups (IN-PRF-EX, IN-CRF-EX, NoT-EX, SOL-EX) in blocks of five trials across the persistence test phase of the experiment. 
tions made by the reformulated learned helplessness theory (Abramson et al., 1978). From this perspective, the demonstrated superiority of partial reinforcement treatment compared with continuous reinforcement treatment in the present experiment is easily understood as a consequence of attributing failure responsibility to unstable factors in the former case. The present findings regarding treatment length seem equally agreeable to an attributional theoretical frame. The greater persistence shown by subjects treated with extended partial reinforcement relative to subjects treated with abbreviated partial reinforcement can be accounted for simply by suggesting the rule that the strength of the attribution (in this case, to unstable events) is linked to the duration of exposure to the conditions that have forced the attribution. Such a rule seems reasonable. It follows that the enhanced persistence associated with extended as opposed to abbreviated continuous reinforcement treatment is a function of the greater strength of the attribution for success to internal, stable factors in the case of extended training, inasmuch as these attributions are also believed to immunize persons against helplessness (Seligman, Abramson, Semmel, \& von Baeyer, 1979).

Our results pose a serious challenge to the idea that previous demonstrations of increased immunization against learned helplessness following partial reinforcement treatment are interpretable within an Amsel-type frustration model (cf. Jones et al., 1977; Nation et al., 1979). Fundamental to the frustration position is the assumption that partial reinforcement enhances persistence by virtue of counterconditioning goal-oriented responding to the emotionally produced (frustrative) cues occasioned by nonreward during training. With continuous reinforcement treatment, no frustration is realized; thus this counterconditioning process is not possible. The belief is that when negative events again present themselves following treatment, as would be the case with protracted episodes of extinction or some other noncontingency, the resulting frustration mediates approach for partial subjects and avoidance for continuous subjects. The difficulty for the frustration model with respect to the present findings is as follows. The theory assumes that an increasing expectancy for reinforcement accompanies increasing treatment length. It is further assumed that the amount of frustration produced by nonreinforcement during treatment is greater, the greater this reinforcement expectancy. Consequently, extended treatment in this study should have occasioned substantially more frustration during the persistence test than did abbreviated treatment. In the instance of continuous reinforcement treatment, this increased frustration effect should have resulted in greater mediated avoidance and less persistence on the part of subjects given extended reversal training; that is, overtraining and extinction effects should have been evident. Obviously, such effects did not occur.

\section{REFERENCE NOTE}

1. Seligman, M. E. P. Personal communication, May 1979.

\section{REFERENCES}

Abramson, L. Y., Seligman, M. E. P., \& Teasdaif, J. D. Learned helplessness in humans: Critique and reformulation. Journal of Abnormal Psychology, 1978, 87, 49-74.

Jones, S. L., Nation, J. R., \& Massad, P. Immunization against learned helplessness in man. Journal of Abnormal Psychology, 1977, 86, 75-83.

Nation, J. R., Cooney, J. B., \& Gartrell, K. E. Durability and generalizability of persistence training. Journal of Abnormal Psychology, 1979, 88, 121-136.

Nation, J. F., \& Massad, P. Persistence training: A partial reinforcement procedure for reversing learned helplessness and depression. Journal of Experimental Psychology: General, $1978,107,436-451$.

Nation, J. R., \& Woods, D. M. Persistence: The role of partial reinforcement in psychotherapy. Journal of Experimental Psychology: General, 1980, 109, 175-207.

Seligman, M. E. P., Abramson, L. Y., Semmel, A., \& von BAEYer, C. Depressive attributional style. Journal of Abnormal Psychology, 1979, 88, 242-247.

Traupmann, K. L. Drive, reward, and training parameters, and the overlearning extinction effect (OEE). Learning and Motivation, 1972, 3, 359-368.

(Received for publication December 4, 1980.) 